\title{
LA GRANJA INTEGRAL AUTOSUFICIENTE: ESTRATEGIA EDUCATIVA DE DESARROLLO SOSTENIBLE EN EL SECTOR RURAL
}

Daniel Herrera Acosta*

\begin{abstract}
This paper s a reflection about the advantages of a farm as educational resource. Also, it presents the idea of the farm as a base of the human development for the Colombian farmer The Universidad Pedagógica Nacional plays an important role in order to seek that the farm could be used as a laboratory where the child finds enough tools to improve his life conditions,

The farm has the property of joining people in order to rise some social values as solidarity, good communication, tolerance, respect, etc. which are the fundamentals of social development.

Besides, the farm lets farmers recognize the level of resources that they have, and doing a good use of them thinking about the future.

Finally it is important to say that the farm will help the child to identify the different changes of energy and how important are those process for keeping his own resources
\end{abstract}

\section{PALABRAS CLAVES}

Desarrollo sostenible, granja autosuficiente, desarrollo humano, estrategia educativa.

\section{INTRODUCCIÓN}

Al reflexionar sobre el papel que pueden cumplir los Licenciados en Biología dentro del panorama educativo del país, cobra cada día más fuerza la necesidad que, desde su formación en la Universidad, se disponga de mecanismos y posibilidades que los habiliten como docentes, capaces de afrontar los retos de una nación con inmensas riquezas naturales y con una población de hombres y mujeres de invaluables calidades humanas e intelectuales.

En efecto, sin mucha dificultad se puede constatar que en el sector rural colombiano existen incontables recursos hídricos, geológicos, de flora y fauna con su correspondiente organización armónica que no justifican, de ninguna manera, la presencia de una población sumida en la pobreza absoluta y con los más bajos indicadores de vida digna.

\footnotetext{
*Profesor asociado Departamento de Biología. Magíster en Educación para la Salud. 
Ante esta dicotomía contradictoria, es necesario rescatar para el desarrollo humano, algunas estrategias accesibles al hombre del campo, que lo animen al progreso individual, familiar y comunitario sin detrimento de su entorno y con el propósito de edificar una patria nueva para si mismo y para sus hijos dentro de principios de sostenibilidad.

Una de ellas puede ser La Granja Integral Autosuficiente, cuyo diseño e implementación en el seno del hogar, puede constituirse, además, en una estrategia educativa que aproxime a la familia a una comprensión práctica y vivencial de la realidad con beneficios para si misma y para su entorno.

Con base en lo anterior, este artículo pretende compartir con los lectores, las tesis que fundamentan la estrategia, para que, desde una perspectiva universal y local, se entienda el significado del desarrollo sostenible y sus efectos, tanto inmediatos como mediatos en el globo que habitamos y en el entorno que vivimos. Por eso, sin pretender agotar la teoría escrita sobre el tema, se desea ponerlo nuevamente sobre el tapete, para analizar sus bondades y limitaciones, pero también, dicho sea de paso, para rescatar de él lo positivo, en procura de mejores condiciones de vida para el hombre del campo.

\section{EL DESARROLLO}

Mucho se ha hablado de lo que significa este término, pero para el efecto que nos ocupa creo que deberíamos empezar por comentar el punto de vista puramente idiomático: "la noción de desarrollo dice relación con rollo, con algo que está arrollado, replegado; y que se desencoge, se despliega. Desarrollar es amplificar, desenvolver, explicar, aumentar, crecer, madurar, progresar, extender. Desarrollo es sinónimo de adelanto, aumento, crecimiento, desenvolvimiento, incremento, maduración, progreso, prosperidad. Hoy también de modernidad"1.

Esta primera disertación lleva implícitas algunas inquietudes como ¿Desarrollo para que? ¿Desarrollo para quiénes? ¿A qué tipo de desarrollo se hará referencia explícita?

Existen dos caras del desarrollo, una exhibida cotidianamente a través de los medios de comunicación, presentándose como logros para el bienestar humano; es tal vez, la cara más evidente. Pero hay otra faceta del desarrollo, un poco más difusa y que sólo se logra comprender por algunos indicadores de su antitesis, esto es, la pobreza. Existen hoy en el mundo dos tipos de pobreza: La tradicional que afecta a una proporción importantísima de la población mundial y la modernizada, como la denomina van Illich, la cual es producto de la modernidad. En efecto, para la pobreza tradicional intervienen variables relacionadas con la inequidad en la distribución de los bienes y/o agresividad y maltrato tecnológico de países altamente industrializados a países en vías de este mismo nivel. Un ejemplo de estas situaciones se ve reflejado en la siguiente cita que hace referencia al libro de J. Chonchol: "Modernisation agricole et exclusions des paysans en Amerique Latine", en la que se contienen las siguientes afirmaciones:

La Revolución Verde, el gran progreso técnico de la agricultura contemporánea, es puesto en cuestión. Casos como México y la India, mostrados en algún momento como ejemplos

\footnotetext{
${ }^{1}$ ELIZALDE Antonio. Modelos de desarrollo e integración económica una mirada crítica desde la perspectiva del sur, CEPAUR, Centro De Alternativas de Desarrollo, CHILE. en Revista, Cuadernos Verdes de Villa de Leyva. Año 4. No.7 mayo de 1993. págs. 8-10
} 
paradigmáticos del éxito de su aplicación en países menos desarrollados, comienzan después a ser fuertemente problematizados por distintas razones: Resultados mucho menos espectaculares en tierras menos buenas; introducción de especies que resultaron débiles frente a las condiciones climáticas; dependencia creciente de los fertilizantes en la medida del debilitamiento de los suelos. Esta modernización suele generar una situación peor para muchos campesinos a través de efectos de mayor dependencia, endeudamiento y hasta su expulsión a las ciudades sin posibilidad clara de un nuevo trabajo, pobreza del atraso.

Pero aún más, para la pobreza de la modernidad se citan como ejemplos las dietas con exceso de proteínas animales que se traducen en enfermedades cardiovasculares; concentración urbana excesiva, que se traduce en estrés y enfermedades respiratorias, entre otras. Porque la pobreza modernizada aparece cuando la intensidad de la dependencia del mercado alcanza un cierto umbral; subjetivamente, quienes sufren la mutilación de una apabullante dependencia de las riquezas de la productividad industrial, experimentan como consecuencia una opulencia frustrante. Simplemente los priva de la libertad y del poder de actuar con autonomía, de vivir creativamente; los confina a la supervivencia por el hecho de estar insertos en las relaciones del mercado. Y precisamente porque padecen esta nueva impotencia de modo tan profundo la expresan con dificultad. Vivimos actualmente un subdesarrollo del crecimiento y a la vez un crecimiento del subdesarrollo, países que se empobrecen a pesar de su creciente riqueza material y un crecimiento absoluto de los pobres tanto de los países pobres como de los países ricos.

"Nuestra sociedad se caracteriza por su casi absoluta incapacidad para distinguir entre crecimiento y desarrollo. Esto se debe a que la ideología del crecimiento es extremadamente atractiva ya que ofrece aún solución a la pobreza sin requerir transformaciones profundas en las actuales formas de distribución de los bienes y servicios y de la regulación del crecimiento de la población"2.

\section{EL DESARROLLO HUMANO}

Aunque existen variadas corrientes ideológicas que orientan a la población humana en procura de su desarrollo, es necesario sin embargo, hacer un análisis de algunas de éstas, con el propósito de ubicar el concepto de desarrollo que más se acerca a la esencia del ser humano como persona, con capacidades intelectuales, espirituales y físicas En efecto Una de las ideas que ha creado escuela a través de la historia de la humanidad es que crecimiento económico es sinónimo de desarrollo humano. Esta concepción que en principio puede tener relaciones de coherencia, no es válida, a nuestro juicio, por cuanto los seres humanos son mucho más que cúmulo de materia, en efecto, el crecimiento cimentado en procesos económicos deja de lado otras características esenciales del ser hombre como son su intelecto, sus sentimientos, sus pasiones, sus emociones, sus procesos de maduración personal, su homeostasia interna y externa, su espiritualidad y su felicidad. Creer que a mayor riqueza material, mayor es el desarrollo humano, es reafirmar el camino para el establecimiento de pocos ricos y muchos pobres, es consolidar la permanencia de las grandes diferencias sociales con mayores oportunidades para unos

\footnotetext{
2 Ibidem
} 
y escasas posibilidades para otros, es negarle al niño, hombre futuro, las potencialidades para madurar, para crear, para conocer, para educarse, para tomar decisiones.

Es además, desvirtuar el concepto del desarrollo humano, ubicándolo como un medio y no como una meta, para que el ser humano encuentre bienestar y felicidad

Otra idea es considerar que el desarrollo humano se logra en la medida que puedan ser satisfechas las necesidades del individuo, tomadas éstas como cambiantes, ilimitadas y siempre crecientes.

Sobre este particular, es conveniente aclarar algunos aspectos; en efecto, durante mucho tiempo se ha creído que las necesidades humanas constituyen una suerte de barril sin fondo, donde cada vez que se "satisface" alguna, inmediatamente surge en su lugar otra nueva, aún más acuciante e indispensable que la anterior. De allí ha derivado una concepción del ser humano como un ser que en forma permanente está tensionado por una necesidad tras otra; y de la economía como el esfuerzo racional para proveer a éste de los bienes y elementos que demanda para colmar sus necesidades.

El error típico que se ha convertido en los análisis acerca de las necesidades humanas es que no se explícita la diferencia esencial entre lo que son propiamente necesidades y lo que son tos satisfactores de esas necesidades. Es indispensable hacer una distinción entre ambos conceptos. Por ejemplo: La alimentación, la vivienda, el abrigo, son satisfactores de la necesidad de subsistencia. Al igual, la educación formal e informal, la investigación y la estimulación precoz, son satisfactores de la necesidad de entendimiento. O los sistemas curativos, la prevención, la higiene y la medicina natural, lo son de la necesidad de protección.

Retomando los trabajos realizados por Manfred Max-Neff, Antonio Elizalde y Martín Hopenhayn, en su libro: "Desarrollo a escala humana: una opción para el futuro", podemos conceptuar por el contrario que el hombre es un ser de necesidades, pero vistas a la vez como carencias y como potencialidades.

Además, las necesidades son pocas, finitas y clasificables, constituyendo un sistema, donde interactúan así mismo los satisfactores y los bienes económicos. Así, los satisfactores son los elementos que cada sistema económico, social y político adopta para la satisfacción de las mismas necesidades humanas. En cada sistema, éstas se satisfacen (o no se satisfacen), a través de la generación (o no generación) de diferentes tipos de satisfactores.

Uno de los aspectos que define a cada cultura es su elección de satisfactores. Las necesidades humanas de un individuo que pertenece a una sociedad consumista son las mismas de aquél que pertenece a una sociedad ascética. Lo que cambia y lo que define el desarrollo humano, en este caso, es la elección de cantidad y calidad de los satisfactores y las posibilidades de tener acceso a los satisfactores requeridos.

\section{EL DESARROLLO SOSTENIBLE}

Continuando con el análisis del significado del Desarrollo, se ha acuñado desde 1980 un concepto nuevo, dándole el calificativo de sostenido o sustentable. De esta manera, en el contexto global de nuestro planeta, un grupo de agencias de las Naciones Unidas, elaboró el concepto, que fue recogido en 1987 por la Comisión Mundial del Medio 
Ambiente y Desarrollo y en el que establece que "para hacer que el Desarrollo sea Sustentable, es necesario asegurar que satisfaga las necesidades del presente, sin comprometer la capacidad de las futuras generaciones para satisfacer las propias" y agrega que el concepto de Desarrollo Sustentable, implica límites, no límites absolutos, sino limitaciones que imponen a los recursos del medio ambiente, el estado actual de a tecnología y de la organización social y la capacidad de la biosfera, de absorber los efectos de las actividades humanas". A renglón seguido, la Comisión advierte que un mundo donde la pobreza es endémica será siempre propenso a sufrir una catástrofe ecológica de uno u otro tipo".

Recogiendo nuevamente el trabajo sobre Desarrollo a escala humana de Max-Neff y Cols., se advierte que ante todo, el Desarrollo Humano se centra en la disponibilidad y elección de satisfactores de calidad para sus necesidades y potencialidades. Si se compara, de otra parte, este análisis con los principios de la Sustentabilidad o Sostenibilidad, se pueden encontrar congruencias valiosas para reiterar la importancia del concepto de Desarrollo dentro de un contexto humano. En efecto, a juicio de Carrizosa Umaña $^{3}$, el Desarrollo Sustentable en Colombia debe consolidarse con estrategias caracterizadas por:

1. Promoción de nuevos valores en las comunidades humanas, como: Solidaridad, Ambientalismo, Liderazgo y Creatividad.

2. Aceleración de la conversión de recursos genéticos en beneficios económicos para las comunidades que los protejan.

3. Democratización de la información y la instrumentalización. La inversión forzosa en investigación y desarrollo de nuevas líneas con ventajas comparativas (biotecnología). Integración de las ciencias sociales con la bioquímica.

4. Recurso a la Energía Biológica. Producción limpia de nuevos bienes tropicales (bioindustria).

5. Democratización de:

- Servicios públicos

- Recreación

—Consumos básicos: proteínas y calorías.

La UICN, Unión Internacional para la Conservación de la Naturaleza, es la institución que ha liderado con las Naciones Unidas, desde 1980, el esfuerzo de definición conceptual de Desarrollo Sostenible: "El Desarrollo es la vía que el pueblo utiliza para satisfacer sus necesidades y mejorar sus vidas. El Desarrollo Sostenible es un proceso de mejoría económica y social, que satisface las necesidades y valores de todos los grupos interesados, manteniendo las opciones futuras y conservando los recursos naturales y la diversidad, es un concepto complejo que incorpora:

1. La sostenibilidad ecológica, es decir el mantenimiento de los procesos ecológicos, la diversidad biológica y los recursos biológicos.

${ }^{3}$ CARRIZOSA UMAÑA, Julio La política ambiental en Colombia. Desarrollo sostenible y democratización FESCOL, Santafé de Bogotá, Colombia, 1992. 
2. La sostenibilidad social, representada por la equidad en la distribución de los bienes individuales y comunitarios.

3. La sostenibilidad cultural, esto es, que el desarrollo sea compatible con la cultura y los valores de los pueblos afectados.

4. La sostenibilidad económica, lo cual hará posible que el desarrollo sea económicamente eficiente y equitativo dentro y entre las generaciones.

Ajuicio del Worldwatch Institute ${ }^{4}$, una pintura de lo que podría ser una sociedad sustentable sería. Energía solar, eficiencia en todos los sentidos, materiales reutilizados 0 reciclados, con una base biológica restaurada y con un nuevo conjunto de valores.

En Colombia, ¿cómo se podría lograr esta utopía? En opinión de Carrizosa, se debería conformar una política de inversión que intente resolver los siguientes problemas:

1. El problema del placer y las substancias psicotrópicas: ¿podría Colombia desarrollar una substancia que satisfaciera la demanda de cambio psicológico sin crear conductas delincuenciales, sin la conformación de adicción y sin consecuencias fisiológicas negativas?

2. El problema de la violencia: ¿cuál es la relación de la violencia con la desnutrición, con la contaminación y con la ausencia de campos de acción?

3. El problema del bajo precio unitario de los productos tropicales: ¿en un país dotado de una de las diversidades biológicas más altas del mundo, cómo transformar esa diversidad en productos que compensen el costo de los que tendremos que seguir importando?

4. El problema de la deficiencia proteínica: ¿cómo asegurar la producción, amplia distribución y consumo de las proteínas fundamentales para el desarrollo cerebral de los ciudadanos colombianos?

\section{UNA ESTRATEGIA EDUCATIVA DE DESARROLLO SOSTENIBLE: LA GRANJA INTEGRAL AUTOSUFICIENTE}

\section{Concepto}

Es un modelo alternativo y diversificado al monocultivo o a la explotación agrícola tradicional del campesino latinoamericano Con su diseño, construcción y ejecución se recuperan las valiosas tradiciones campesinas y se complementan con conocimientos de ecología, control biológico, y conservación de recursos primarios como suelos, clima y agua. Además, permite su transformación y la aplicación de tecnologías apropiadas a las condiciones del medio, a los recursos de la familia y a la articulación a los mercados y dinámica agropecuaria local.

\footnotetext{
${ }^{4}$ BROWN, Lester. State of the World Worldwatch lnstitute. Norton New York, 1990.
} 


\section{Aportes para el desarrollo humano sostenible}

El lema de trabajo que la Organización Mundial de la Salud OMS, propuso en 1989 para afrontar los problemas de salud de las comunidades humanas fue:

"Pensad Globalmente, Actuad Localmente", sin duda alguna este principio de acción también se puede aplicar en este contexto, porque de nada sirven las reflexiones teóricas sobre el significado del desarrollo a nivel universal, si no se buscan mecanismos locales para hacerlos prácticos a la realidad que vivenciamos cada día Es por esto, que el presente ensayo toma como núcleo de reflexión-acción, en la familia campesina colombiana, la granja integral autosuficiente, para aproximar a través de ella al Lector a la comprensión de lo que ésta puede aportar para un desarrollo humano sostenible. En efecto, se puede demostrar, que ésta, puede ser una estrategia educativa al alcance del hombre del campo, que lo habilita para:

1 Promover valores de solidaridad, liderazgo y creatividad en su hogar.

2 Hacer tangibles los ciclos de conservación de la energía mediante las cadenas tróficas presentes en la granja, y que se constituyen en fundamentos teóricos esenciales de producción y conservación de los recursos naturales para beneficio de Las generaciones presentes y futuras.

3. Aproximarse a la tecnología, cada vez más en aumento, del uso de la energía solar en sus mejores niveles de eficiencia, con materiales reutilizados y/o reciclados que hacen comprensible lo que significa una base biológica restaurada con un nuevo conjunto de valores.

4. Establecer lazos estrechos de comunicación entre la escuela y la familia. Significa esto, que poco a poco podrá transformar la dicotomía divergente que existe entre los saberes aprendidos en la escuela y los que forman parte de la cotidianidad en la parcela, por una relación convergente que alimente la comprensión de la vida y todo lo que ésta encierra y trasciende para el desarrollo humano.

5. Generar actitudes positivas hacia la investigación experimental, las cuales permiten una democratización de la información y la instrumentalización. En efecto, la granja es un libro abierto de conocimientos, para cada uno de los miembros de la familia. Es además el mejor laboratorio de ensayos y experimentos que pueda tener un maestro de Biología para demostrar las bondades y maravillas de la naturaleza a sus alumnos.

6. Proponer formas alternativas de progreso económico y social para las familias que constituyen una Comunidad Educativa Esto se hace evidente, cuando suma sus esfuerzos a aquellos de otras familias, constituyendo cooperativas que posibilitan nuevos horizontes para mejorar sus condiciones de vida.

7. Recuperar la credibilidad en sus capacidades como persona y en los invaluables recursos que posee, ya sean estos del orden natural, social y/o creado y lograr con ello, sentimientos de identidad y pertenencia con su terruño.

8. Fomentar la construcción de ambientes de trabajo solidario entre la comunidad, para facilitar procesos de tolerancia y derrotar acciones, algunas veces endémicas, de violencia entre las poblaciones humanas. 
9 Asegurar fuentes directas de nutrientes para la familia en situaciones controladas técnicamente, que garantizan una alimentación balanceada para cada uno de sus miembros

10 Contribuir mediante acciones locales a una sensibilización comunitaria frente al pensamiento global del desarrollo humano sostenible

\section{FLUJO DE RELACIONES ENTRE LOS ELEMENTOS Y PROCESOS DE LA GRANJA INTEGRAL AUTOSUFICIENTE}

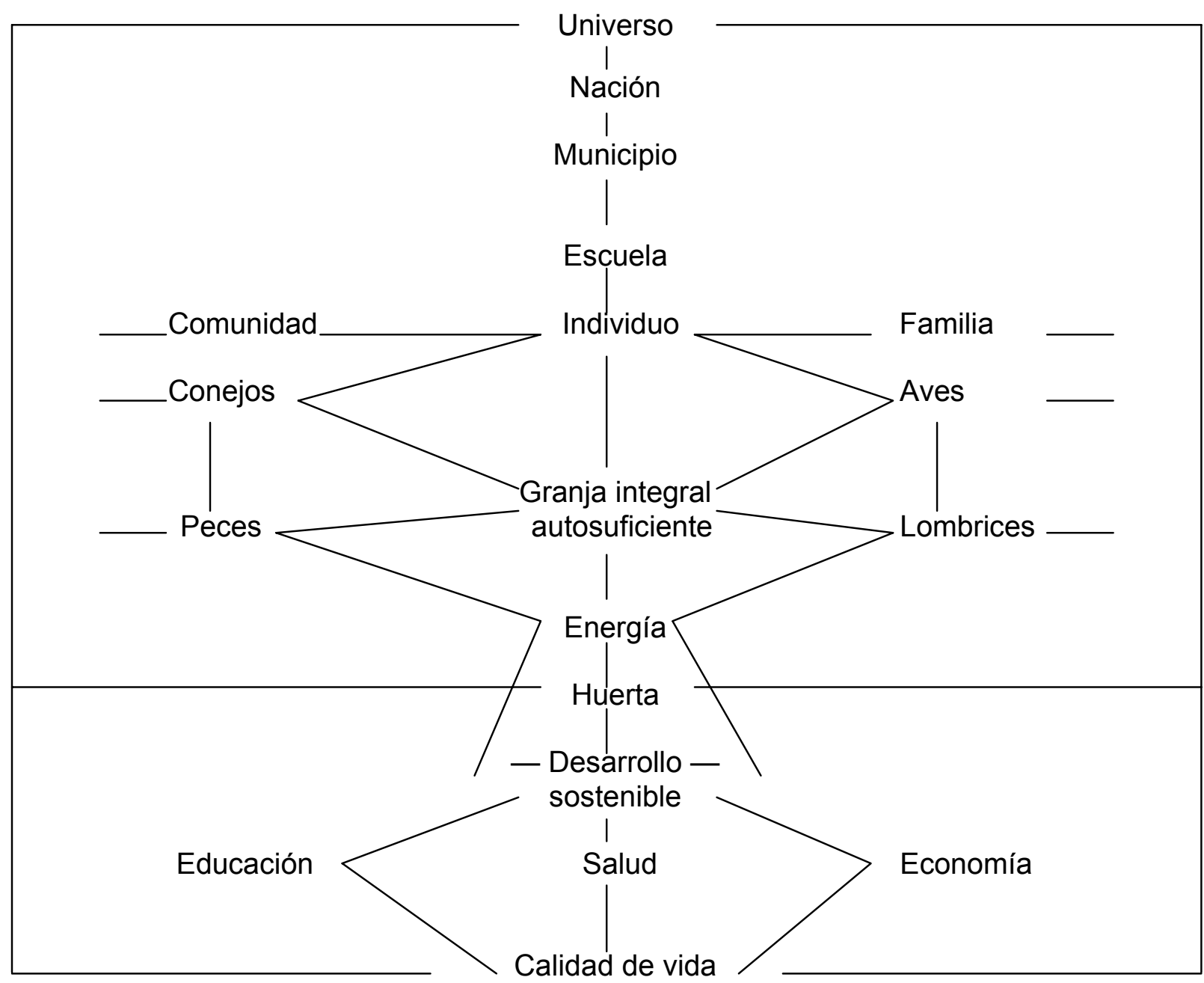

\section{BIBLIOGRAFÍA}


1. ANGARITA BARON. Ciro El desarrollo sostenible y la Constitución de 1991, en Revista DIVERSA, revista de pensamiento ambiental. Ministerio del Medio Ambiente, primer trimestre de 1996, pág. 46.

2 BROWN. Lester. State of the World. Worldwatch Instítute Norton. New York, 1990.

3 CARRIZOSA UMAÑA, Julio. La política ambiental en Colombia: Desarrollo sostenible y democratización FESCOL, Santafe de Bogota. Colombia, 1992.

4. CARRIZOSA UMAÑA, Julio. Hacia una cultura de la sostenibilidad En Revista DIVERSA, Bogotá, M.M.A., primer trimestre de 1996, pág. 29

5. CONFERENCIA DE LAS NACIONES UNIDAS SOBRE EL MEDIO AMBIENTE Y EL DESARROLLO, Río de Janeiro, 3 a 14 de junio de 1992, FESCOL.

6. DÁVILA RICCIARDI, Francisco. El sector rural, base para la reconstrucción de grandes propósitos nacionales. En Revista Nacional de Agricultura, SAL, Bogotá, 1997

7. ELIZALDE, Antonio. Modelos de desarrollo e integración económica, una mirada critica desde la perspectiva del sur. CEPAUR, Centro De Alternativas de Desarrollo. CHILE, en Revista, Cuadernos Verdes de Villa de Leyva, Año 4, No. 7, mayo de 1993.

8. HOGARES JUVENILES CAMPESINOS. Manual de la granja, Biblioteca del Campo, La Granja Integral Autosuficiente. 1996.

9. MAX-NEEFF. Manfred, ELIZALDE, Antonio y otro. Desarrollo a escala humana: una opción para el futuro, Uppsala, 1986.

10. MINISTERIO DE EDUCACION NACIONAL, Lineamientos generales para una política nacional de educación ambiental. Bogotá, 1995.

11. TALERO DE HUSAIN, Elsa; UMAÑA DE GAUTHIER, Gloria. Aportes para la realización del Proyecto de Educación Ambiental. Bogotá, 1998,

12. VALENCIA BUITRAGO, Jorge E. Una estrategia de educación ambiental, Programa Teleiworkshop. UPTC, Tunja, 1995. 